\title{
Dynamic Fixation Techniques for the Prevention of Adjacent Segment Disease: A Retrospective Controlled Study
}

\author{
Salvador Fuster ${ }^{1}$, Jaime Jesús Martínez-Anda ${ }^{1}$, Sergio Antonio Castillo-Rivera ${ }^{2}$, \\ Caribay Vargas-Reverón ${ }^{1}$, Eduard Tornero ${ }^{1}$ \\ ${ }^{1}$ Spine Surgery Department, Hospital Clinic of Barcelona, Universitat de Barcelona, Barcelona, Spain \\ ${ }^{2}$ Spine Surgery Department, Hospital Doctor Sótero del Río, Santiago, Chile
}

Study Design: Retrospective, controlled study.

Purpose: Dynamic fixation (topping-off technique) adjacent to a transforaminal lumbar interbody fusion (TLIF) level was developed to reduce the risk of adjacent segment disease (ASDi). This study was designed to compare the clinical and radiological outcomes between patients who underwent circumferential lumbar fusion (CLF) without the topping-off technique, CLF with dynamic rod constructs (DRC), and CLF with interspinous device (ISD).

Overview of Literature: Lumbar fusion can result in the re-distribution of stress, increased mobility, and increased intradiscal pressure at adjacent levels, ultimately leading to adjacent segment degeneration (ASDe) and ASDi. Dynamic fixation techniques (topping-off techniques) adjacent to vertebral fusion have been developed to reduce the risk of ASDe and ASDi because they provide a transitional zone between a caudal rigid fused segment and cephalad-mobile unfused levels.

Methods: A single-center, retrospective, controlled study was designed, including all patients who underwent CLF due to degenerative lumbar spinal disease in Hospital Clinic of Barcelona between 2012 and 2018. Three groups of patients were evaluated as per the type of topping-off technique used: CLF alone group, DRC group, and ISD group. Clinical and radiological outcomes were evaluated. Results: A total of 117 patients were enrolled in the study. Sixty patients (51.3\%) underwent CLF without dynamic stabilization, 24 (20.5\%) were treated with DRC as topping-off technique, and 33 (28.5\%) were treated with an ISD. A total of 12 patients $(20.0 \%)$ in the CLF alone group showed ASDi at the final follow-up, compared to $1(4.2 \%)$ in the DRC group $(p=0.097)$ and $2(6.1 \%)$ in the ISD group ( $p=0.127$ ). The Cox regression model identified a significantly decreased risk of ASDi when a topping-off technique (DRC or ISD) was used (hazard ratio, 0.154; 95\% confidence interval, 0.31-0.77).

Conclusions: Dynamic fixation adjacent to CLF was a safe and efficient procedure associated with improved clinical outcomes in patients with lumbar spine degenerative disease.

Keywords: Lumbosacral region; Lumbar instrumentation; Adjacent segment degeneration; Posterolateral fusion

Received Nov 13, 2020; Revised Jan 29, 2021; Accepted Jan 31, 2021

Corresponding author: Eduard Tornero

Spine Surgery Department, Hospital Clínic of Barcelona, C/Villarroel 170, 08036 Barcelona, Spain

Tel: +34-932275533, Fax: +34-934514438, E-mail: etornero@clinic.cat 


\section{Introduction}

Stiffness caused by lumbar fusion can result in the redistribution of stress, increased mobility, and increased intradiscal pressure at adjacent levels; these biomechanical changes can lead to the development and progression of adjacent segment degeneration (ASDe) and adjacent segment disease (ASDi) $[1,2]$. However, some questions remain unsolved on this matter, such as the extent to which the ASDi represents the natural history of disease, how lumbar fusion interacts as a risk factor, or which current surgical techniques protect against ASDi.

Dynamic fixation techniques (topping-off techniques) adjacent to vertebral fusion have been developed to reduce the risk of ASDe and ASDi in cases where lumbar fusion is needed. The rationale of these topping-off techniques is that they provide a transitional zone between a caudal rigid fused segment and cephalad-mobile unfused levels that may decrease the incidence of ASDi [3].

This study aimed to compare the clinical and radiological outcomes of patients who underwent transforaminal lumbar interbody fusion (TLIF) alone (only fusion, OF) (Fig. 1A), and two different topping-off techniques, hybrid transition system with dynamic rod constructs (DRC) (Fig. 1B) and interspinous device (ISD) (Fig. 1C) were used in patients with radiological proven initial disc degeneration adjacent to the fusion level.

\section{Materials and Methods}

\section{Patients}

We retrospectively reviewed all the patients who were diagnosed with degenerative lumbar spinal disease and underwent TLIF with posterolateral instrumentation, with or without additional dynamic instrumentation of the adjacent superior segment to the TLIF level (toppingoff technique) between January 2012 and December 2018 in Hospital Clinic of Barcelona. For the present study, the following inclusion criteria were applied: (1) axial pain or radicular symptomatology of lumbar spine origin, (2) single- or two-level lumbar fusion, (3) intervertebral disc degeneration of adjacent segment to the fusion surgery $\geq 2$ as per the UCLA grading scale for intervertebral disc degeneration [4] or Pfirrmann [5] $\geq 2$ on magnetic resonance imaging (MRI), and (4) unsuccessful conservative therapy for $>6$ months. Patients with preoperative Oswestry Dis- ability Index (ODI) $\leq 20$, prior history of osteoporosis or metabolic bone disease, lumbar vertebral fracture adjacent to fusion levels, or follow-up $<24$ months were excluded. We recorded data regarding demographic characteristics (age and sex) and body mass index (BMI). This study was performed as per the ethical standards of the research committees at the Hospital Clinic of the University of Barcelona and the 1964 Helsinki Declaration and its later amendments or comparable ethical standards. Informed consent was obtained from all the enrolled subjects. The institutional ethics and review boards approved the study (register no., $\mathrm{HCB} / 2020 / 0674$ ).

\section{Clinical and radiological evaluation}

Clinical preoperative evaluation and surgical outcomes were evaluated using pre- and postoperative ODI [6] and Visual Analog Scale (VAS) for lumbar and radicular pain. Data were collected preoperatively; 1, 3, 6, and 12 months postoperatively; and yearly thereafter. Data regarding surgical complications and reoperation rates were also reviewed.

All the patients were preoperatively assessed using standing and dynamic lumbar radiography and lumbar MRI with 1.5T equipment. For postoperative evaluations, lumbar radiographs were obtained at every followup consultation. MRI was performed only in cases of symptom recurrence. Adjacent segment was considered the segment superior to the fused level or the segment included with dynamic instrumentation if the topping-off technique was used; supra-adjacent segment was considered the lumbar segment superior to the adjacent segment (Fig. 1A). Pfirrmann [5] and Modic [7] scales were used for analyzing preoperative lumbar disc degeneration and endplate changes, respectively. Lumbar lordosis (L1-S1 Cobb's angle), pelvic incidence, pelvic tilt, sacral slope, segmental angulation (segmental Cobb's angle), intersomatic disc height, and UCLA grade for intervertebral disc degeneration were measured preoperatively and during the follow-up period in affected, adjacent, and supraadjacent segments [4]. The criteria for postoperative diagnosis of ASDe in adjacent and supra-adjacent segments were as follows: (1) disc height decrease $\geq 25 \%$, (2) disc wedging $>5^{\circ}$, and (3) worsening in UCLA grading scale for intervertebral disc degeneration [8,9]. A diagnosis of ASDi was established in patients with proven radiological progression of ASDe and worsening of follow-up ODI 
$>30$ or lumbar/radicular pain with VAS score $>5$ at the final clinical evaluation.

\section{Surgical technique}

During surgery, interbody cages with autogenous bone grafting were used for intervertebral fusion. The $\mathrm{N}$-flex (Synthes Spine Inc., West Chester, PA, USA) system was used for posterior transpedicular instrumentation and Stenofix (DePuy Synthes Inc., Oberdorf, Switzerland) was used for interspinous instrumentation. Laminectomy with foraminal decompression, facetectomy, discectomy, and interbody fusion were performed using the conventional TLIF procedure, followed by pedicle screw fixation. When dynamic rods were used, screws were placed lateral to the facet joints. The bumper cord at the non-fusion dynamic stabilization segment and titanium rod at the fusion segment were connected using the $\mathrm{N}$-flex system (Synthes Spine Inc.). In cases of ISD surgery, it was situated optimally in the spinous processes; handheld compression instruments were used to clamp the plates toward each other, closing the system and driving the spikes into the bone. Follow-up evaluations were performed on the first 10 days after hospital discharge; at 1,3, and 6 months after discharge; and each year thereafter.

\section{Statistical analyses}

Continuous variables are expressed as mean and standard deviation (SD) or median and interquartile range (IQR) values and were compared using the Student $t$-test or the Mann-Whitney $U$-test as per the Kolmogorov-Smirnov test of normality. Qualitative variables were described using absolute frequencies and percentages and were compared using the chi-square test or Fisher's exact test, when necessary. McNemar test and Wilcoxon test were applied for continuous and qualitative variables, respectively, for comparing paired preoperative and postoperative data. The Kaplan-Meier hazard method was used to estimate the cumulative probability of ASDi within 5 years of surgery. The log-rank test was applied to compare hazard curves. A logistic regression model was used to identify independent variables associated with ASDe. The goodness-of-fit was explored based on the Hosmer-Lemeshow test. A Cox regression model was performed to identify independent variables associated with ASDi. The presence of interaction and the role of confounding factors were evaluated. Statistical significance was defined as a two-tailed $p<0.05$. The analysis was performed using IBM SPSS ver. 20.0 (IBM Corp., Armonk, NY, USA).

\section{Results}

A total of 117 patients were enrolled. The average (SD) age of the cohort was $57.3 \pm 12.5$ years, and $56.4 \%$ (66) were women. A total of 57 patients (48.7\%) underwent single-level fusion (L5-S1 in 21 cases, L4-L5 in 34 cases, and L3-L4 in two cases) and 60 (51.3\%) underwent two-level fusion (L4-S1 in 43 cases, L3-L5 in eight cases, and L2L4 in nine cases). The mean (SD) BMI of the cohort was $27.57 \mathrm{~kg} / \mathrm{m}^{2}$, and $48(41.0 \%)$ of the patients were smokers. Lumbar pain (20 cases, 17.1\%), radicular symptoms (13 cases, $11.1 \%$ ), or both (84 cases, $71.8 \%$ ) were present for a median period of 24 months (IQR, $20-40$ months) before the surgery. Preoperative median ODI and VAS score were 42 (IQR, 38-48) and 7 (IQR, 6-8), respectively. The Pfirrmann preoperative classification of disc degeneration at the index, adjacent, and supra-adjacent level were $>3$ in $110(94.0 \%), 20(17.1 \%)$, and 5 (4.3\%) patients, respectively [5], and that at the supra-adjacent level showed arthritic disc changes $\geq 2$ as per the UCLA grade scaling in 34 patients (29\%). Spondylolisthesis was present in 60 cases (51.3\%) with a median of $15 \%$ (IQR, 10\%-23.75\%) in percentage of listhesis. Modic changes before the surgery were present in 84 cases (71.8\%) [7]. The mean (SD) disc height at the adjacent and supra-adjacent levels before the surgery was $10.3 \pm 2.3 \mathrm{~mm}$ and $10.1 \pm 2.1 \mathrm{~mm}$, respectively. The mean (SD) L1-S1 Cobb's angle, pelvic incidence, pelvic tilt, and sacral slope were $50.5^{\circ} \pm 11.4^{\circ}$, $59.6^{\circ} \pm 11.4^{\circ}, 23.1^{\circ} \pm 7.9^{\circ}$, and $36.7^{\circ} \pm 8.4^{\circ}$, respectively. Sixty patients (51.3\%) underwent TLIF lumbar fusion alone (OF group) without dynamic stabilization, while 24 (20.5\%) were treated with DRC as the topping-off technique (DRC group) and 33 (28.5\%) were treated with ISD (ISD group). The baseline characteristics of patients and outcomes as per the type of surgical treatment are shown in Table 1.

The average preoperative and final follow-up L1-S1 Cobb's angle ( $50.5^{\circ}$ versus $49.9^{\circ}, p=0.60$ ), pelvic incidence (59.9 versus $\left.59.6^{\circ}, p=0.88\right)$, pelvic tilt $\left(23.1^{\circ}\right.$ versus $24.2^{\circ}$, $p=0.35$ ), and sacral slope ( $36.7^{\circ}$ versus $36.2^{\circ}, p=0.61$ ) as per the type of treatment; there was a significant difference in the OF versus DRC groups in SS $\left(37.9^{\circ}\right.$ versus $34.2^{\circ}, p=0.032$ ) (Fig. 2). After a median follow-up of 37 months (IQR, 33.5-45.5 months), 80 patients (68.4\%) 

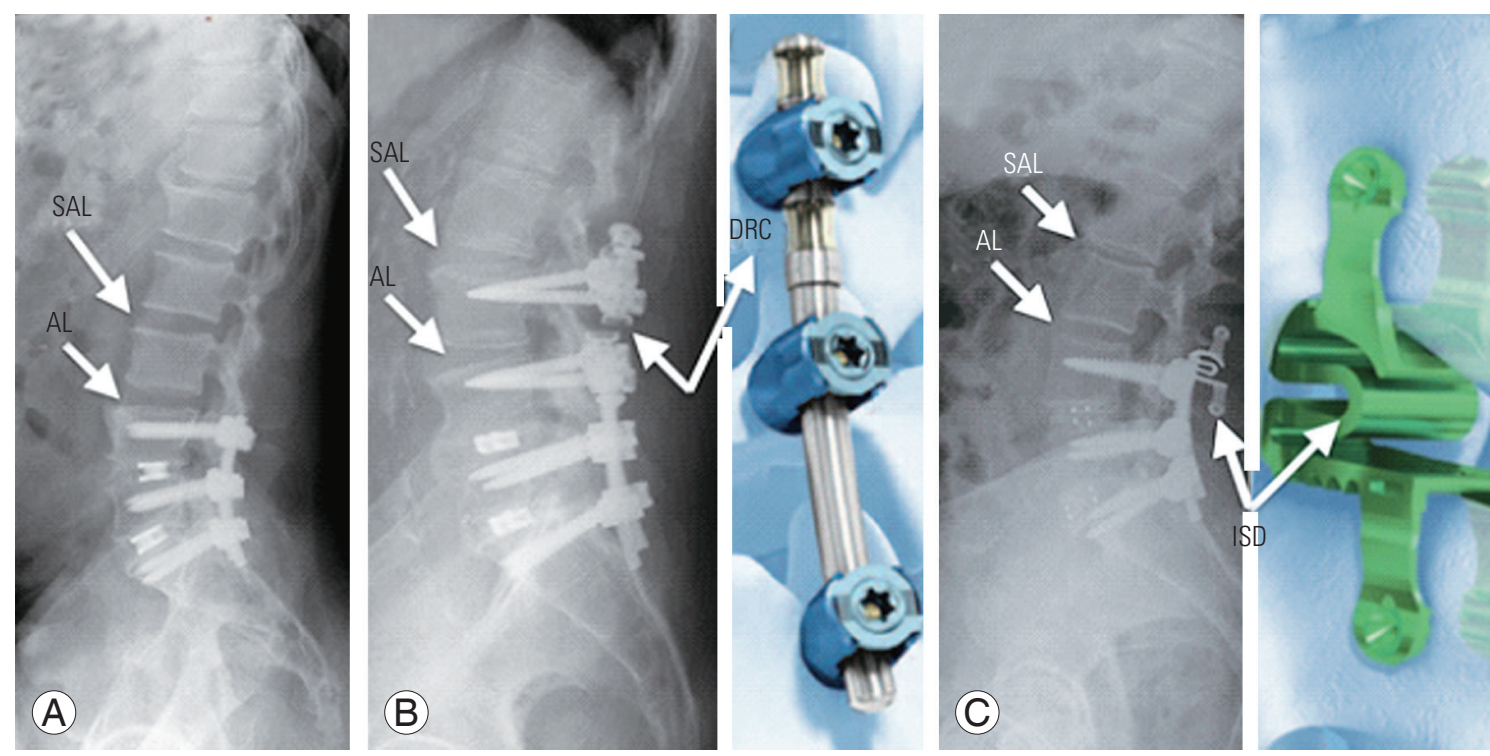

Fig. 1. (A) Only fusion technique. (B) Dynamic fixation as topping-off technique with dynamic rods construct (N-flex, copyright from NFLEX Brochure-DePuySynthes Technique guide). (C) Dynamic fixation as topping-off technique with interspinous device (Stenofix, copyright from Stenofix DePuy Synthes Technique guide). AL, adjacent level; SAL, supra-adjacent level; DRC, dynamic rods construct; ISD, interspinous device.
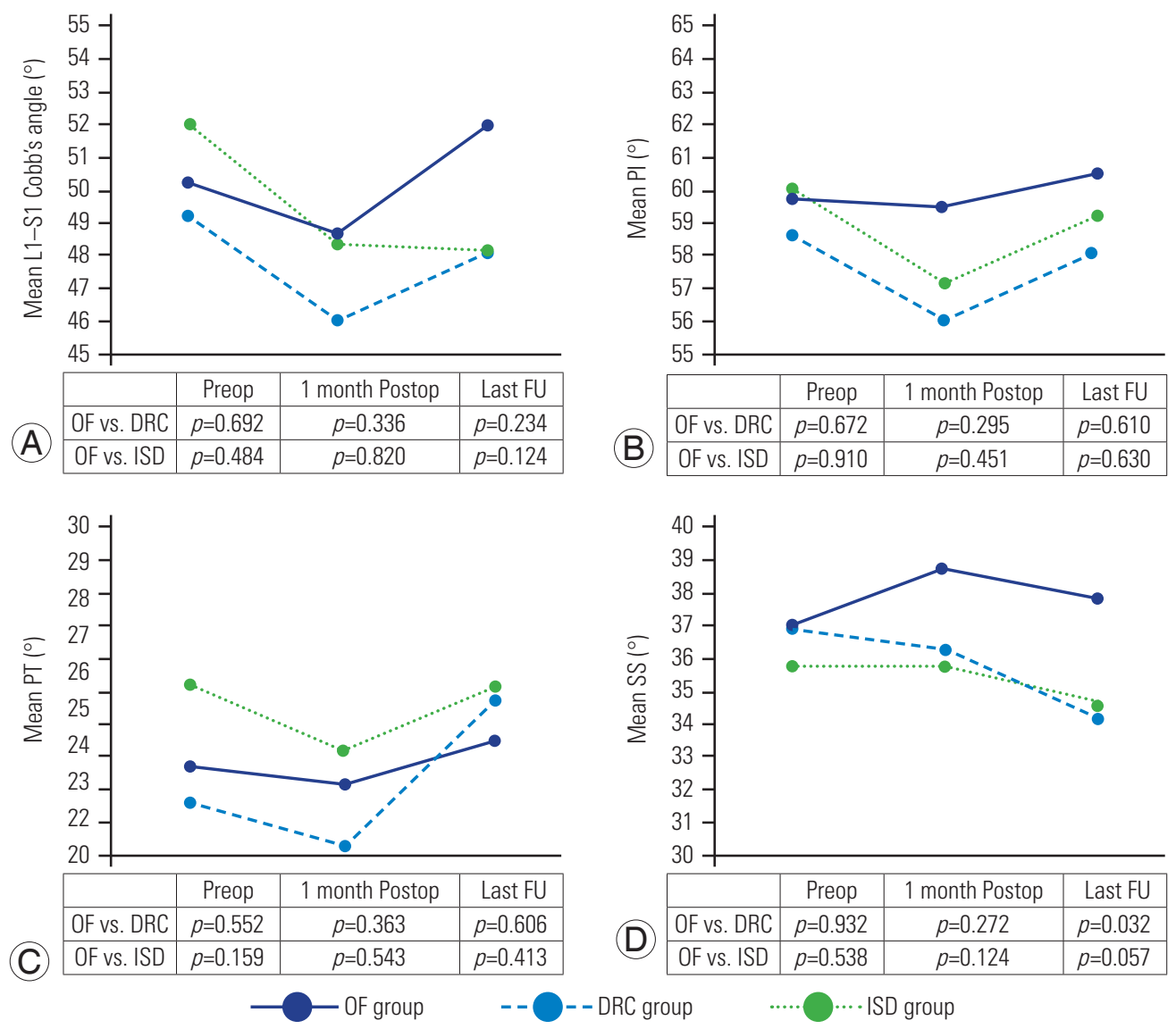

Fig. 2. Mean L1-S1 Cobb's angle (A), pelvic incidence (PI) (B), pelvic tilt (PT) (C), and SS (D) measured prior to surgery, 1 month postoperative (Postop), and at last follow-up (FU), according to group of treatment: only fusion (OF group), dynamic rods construct (DRC group), and interspinous device (ISD group). Preop, preoperative. 

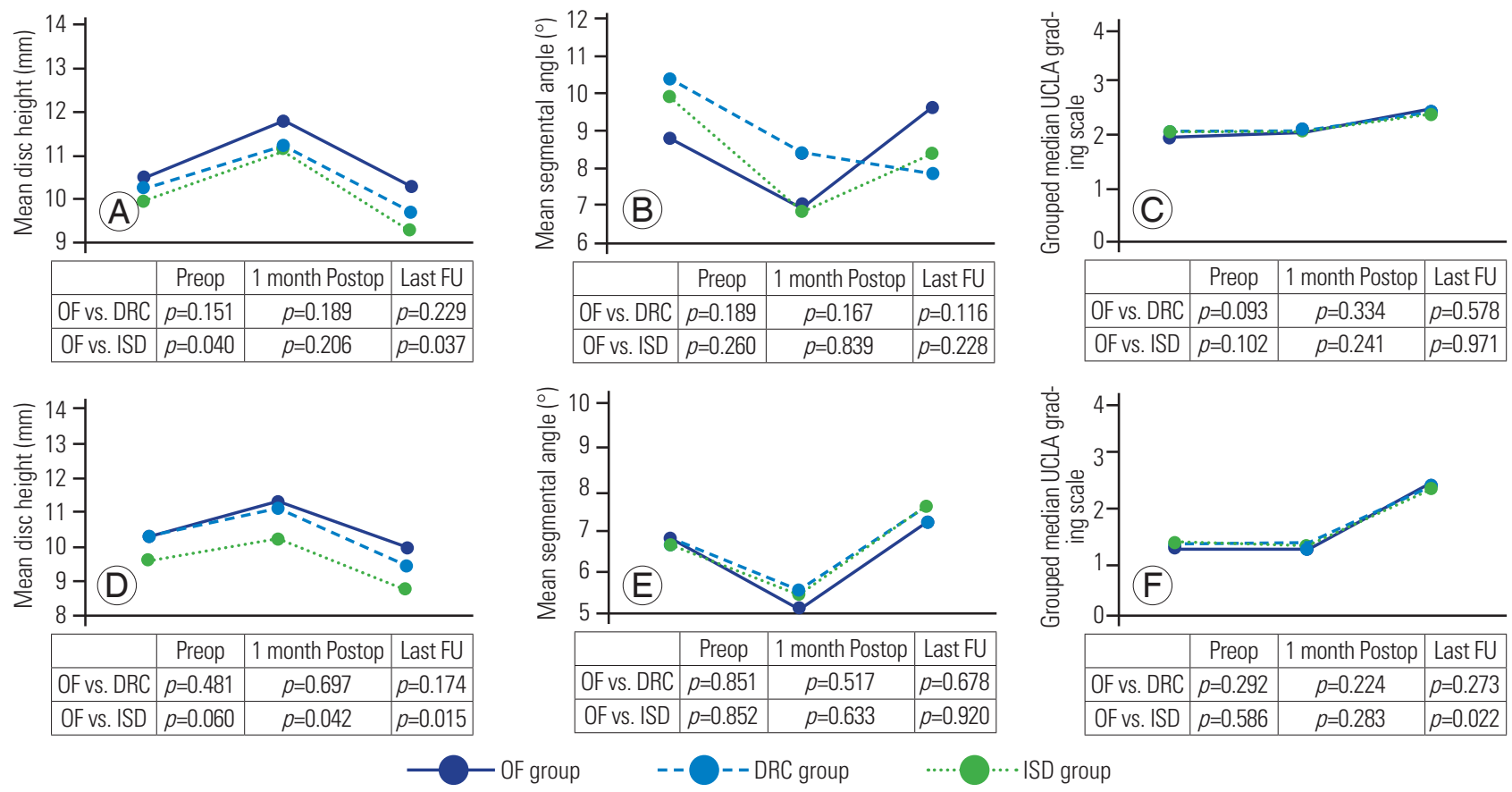

Fig. 3. Mean disc height (A, D), mean adjacent segmental angle (B, E), and grouped median UCLA grading scale (C, F) of adjacent and supra-adjacent segment, measured prior to surgery, 1 month postoperative (Postop), and at last follow-up (FU), according to group of treatment: only fusion (OF group), dynamic rods construct (DRC group), and interspinous device (ISD group). Preop, preoperative.

showed progression of ASDe and 15 (12.8\%) developed ASDi (as per the criteria defined in the "Methods" section). Fig. 3 shows the previously defined ASDe criteria (mean disc height, mean segmental angle, and grouped median UCLA grading scale) before the surgery, 1 month postoperatively, and at the final clinical evaluation as per the group of treatment in the adjacent and supra-adjacent segment; there was a significant difference in the disc height at the supra-adjacent level between the OF and ISD groups during follow-up (10 mm versus $8.7 \mathrm{~mm}$, $p=0.015$ ), with a greater disc height decrease in the ISD group and a greater progression in the UCLA scale at the supra-adjacent level in the ISD group than that in the OF group. The medium preoperative and final follow-up VAS score ( 7 and 2, $p<0.001)$ and ODI (42 and 26, $p<0.001)$ as per the treatment group, as shown in Fig. 4 , with a significant deterioration in the clinical status at the OF group versus DRC or ISD in both the scales. Fig. 5 shows the cumulative hazard function of ASDi within 60 months of surgery as per the use of a topping-off technique (DRC or ISD). There were no complications associated to toppingoff constructs, such as instrument loosening.

All the variables studied and potentially associated with ASDe and ASDi were included in the multivariate analysis
(Table 2). The enter logistic regression model only identified as independent variable associated with ASDe the Pfirrmann preoperative classification of disc degeneration at the adjacent level (odds ratio, 5.323; 95\% confidence interval [CI], 1.06-26.9) [5]. The enter Cox regression model identified a significant decreased risk of ASDi when a topping-off technique was used (hazard ratio, 0.154; 95\% CI, 0.31-0.77).

\section{Discussion}

ASDe involves a series of arthritic changes that occur in vertebral segments cranial and caudal to a lumbarinstrumented fusion; in contrast, ASDi is a clinical condition that causes lumbar pain and radicular symptoms attributable to these ASDe changes [2]. ASDe of the cranial level develops in up to $80 \%$ of the subjects after lumbar fusion. Previous studies have reported a variable correlation between ASDe and clinical outcome $[1,8,10]$. ASDi ranges from $5.2 \%$ to $36.1 \%$ at the 10 -year follow-up after instrumented fusion $[3,9]$; when conservative treatment fails in patients with ASDi, revision surgery is required in up to $36 \%$ of the cases, with poor results and progression to failed back syndrome [11]. 
Table 1. Baseline characteristics of patients and outcomes according to group of treatment: OF, DRC, or ISD groups

\begin{tabular}{|c|c|c|c|c|c|c|}
\hline Characteristic & OF group ( $n=60$ ) & DRC group ( $n=24)$ & $p$-value ${ }^{\text {a) }}$ & ISD group ( $n=33$ ) & $p$-value ${ }^{\text {b) }}$ & $p$-value ${ }^{c)}$ \\
\hline \multicolumn{7}{|l|}{ Baseline characteristics of patients } \\
\hline Age (yr) & $54.0 \pm 13.4$ & $59.5 \pm 11.0$ & 0.078 & $61.7 \pm 9.9$ & 0.005 & 0.441 \\
\hline Female & $36(60.0)$ & $9(37.5)$ & 0.062 & $21(63.6)$ & 0.730 & 0.051 \\
\hline Body mass index $\left(\mathrm{kg} / \mathrm{m}^{2}\right)$ & $28.0 \pm 4.6$ & $29.2 \pm 2.7$ & 0.132 & $25.6 \pm 3.7$ & 0.013 & $<0.001$ \\
\hline Smokers & $35(58.3)$ & $12(50.0)$ & 0.487 & $22(66.7)$ & 0.430 & 0.205 \\
\hline Symptoms prior to surgery & & & $0.590^{d)}$ & & $0.419^{d)}$ & $0.677^{\mathrm{d})}$ \\
\hline Lumbar pain & $10(16.7)$ & $3(12.5)$ & & $7(21.2)$ & & \\
\hline Radicular symptoms & $9(15.0)$ & $2(8.3)$ & & $2(6.1)$ & & \\
\hline Both lumbar and radicular & 41 (68.3) & $19(79.2)$ & & $24(72.7)$ & & \\
\hline Duration with symptoms $(\mathrm{mo})$ & $24(16-40)$ & $30(22-46)$ & 0.292 & $30(23-36)$ & 0.170 & 0.980 \\
\hline Type of vertebral fusion & & & 0.084 & & 0.080 & 0.885 \\
\hline Single fusion & $35(58.3)$ & $9(37.5)$ & & $13(39.4)$ & & \\
\hline Two-level fusion & $25(41.7)$ & $15(62.5)$ & $0.402^{e)}$ & $20(60.6)$ & $0.016^{\mathrm{e})}$ & $0.463^{\mathrm{el}}$ \\
\hline L5-S1 & $13(21.7)$ & $3(12.5)$ & & $5(15.2)$ & & \\
\hline L4-L5 & $21(35.0)$ & $6(25.0)$ & & $7(21.2)$ & & \\
\hline L3-L4 & $1(1.7)$ & 0 & & $1(3.0)$ & & \\
\hline L4-S1 & $18(30.0)$ & $11(45.8)$ & & $14(42.4)$ & & \\
\hline L3-L5 & $6(10.0)$ & $2(8.3)$ & & 0 & & \\
\hline L2-L4 & $1(1.7)$ & $2(8.3)$ & & $6(18.2)$ & & \\
\hline \multicolumn{7}{|c|}{ Pffirmann preoperative classification $>3$ at } \\
\hline Index level & $54(90.0)$ & $23(95.8)$ & 0.667 & $33(100.0)$ & 0.086 & 0.421 \\
\hline Adjacent level & $6(10.0)$ & $7(29.2)$ & 0.043 & $7(21.2)$ & 0.210 & 0.491 \\
\hline Supra-adjacent level & 0 & $3(12.5)$ & 0.021 & $2(6.1)$ & 0.123 & 0.396 \\
\hline Spondylolisthesis prior to surgery & $37(61.7)$ & $10(41.7)$ & 0.095 & 13 (39.4) & 0.039 & 0.863 \\
\hline Listhesis $(\%)^{\eta}$ & $18(10.0-30.0)$ & $12.5(8.0-16.5)$ & 0.102 & $10(10.0-13.5)$ & 0.056 & 0.823 \\
\hline Modic changes $>1$ prior to surgery & $45(75.0)$ & $12(50.0)$ & 0.027 & $27(81.8)$ & 0.045 & 0.011 \\
\hline \multicolumn{7}{|l|}{ Outcomes } \\
\hline Follow-up (mo) & $38(32-45)$ & $35(29-36)$ & 0.002 & $42(35-71)$ & 0.010 & $<0.001$ \\
\hline Bleeding during surgery $(\mathrm{mL})^{g)}$ & $442 \pm 327$ & $279 \pm 144$ & 0.049 & $397 \pm 256$ & 0.644 & 0.164 \\
\hline Duration of surgery (min) & $223 \pm 57$ & $236 \pm 91$ & 0.524 & $217 \pm 45.3$ & 0.607 & 0.360 \\
\hline Fusion achieved & $60(100.0)$ & $24(100.0)$ & - & $33(100.0)$ & - & - \\
\hline ASDe at last follow-up & $38(63.3)$ & $16(66.7)$ & 0.773 & $26(78.8)$ & 0.124 & 0.305 \\
\hline ASDi at last follow-up & $12(20.0)$ & $1(4.2)$ & 0.097 & $2(6.1)$ & 0.127 & 1.000 \\
\hline Required new surgery due to ASDi & $3(5.0)$ & $1(4.2)$ & 1.000 & $1(9.1)$ & 0.931 & 0.618 \\
\hline
\end{tabular}

Values are presented as mean \pm standard deviation, number (\%), or median (interquartile range), unless otherwise stated. Statistically significant differences are shown in bold.

OF group, only fusion group; DRC group, dynamic rods construct group; ISD group, interspinous device group; ASDe, adjacent segment degeneration; ASDi, adjacent segment disease (according to criteria defined in methods section).

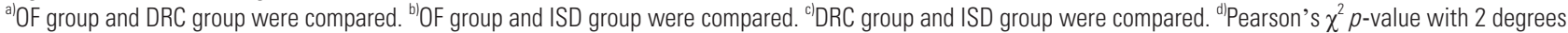
of freedom to test the data distribution of all type of symptomatology. ${ }^{\text {elPearson's }} \chi^{2} p$-value with 5 degrees of freedom to test the data distribution of all type of vertebral fusion. $\left.{ }^{\text {flC} C a l c u l a t e d ~ a m o n g ~ p a t i e n t s ~ w i t h ~ s p o n d y l o l i s t h e s i s ~(~} n=60\right) . ~{ }^{9}$ Data available in 23 (OF group), 12 (DRC group), and 16 (ISD group) cases. 

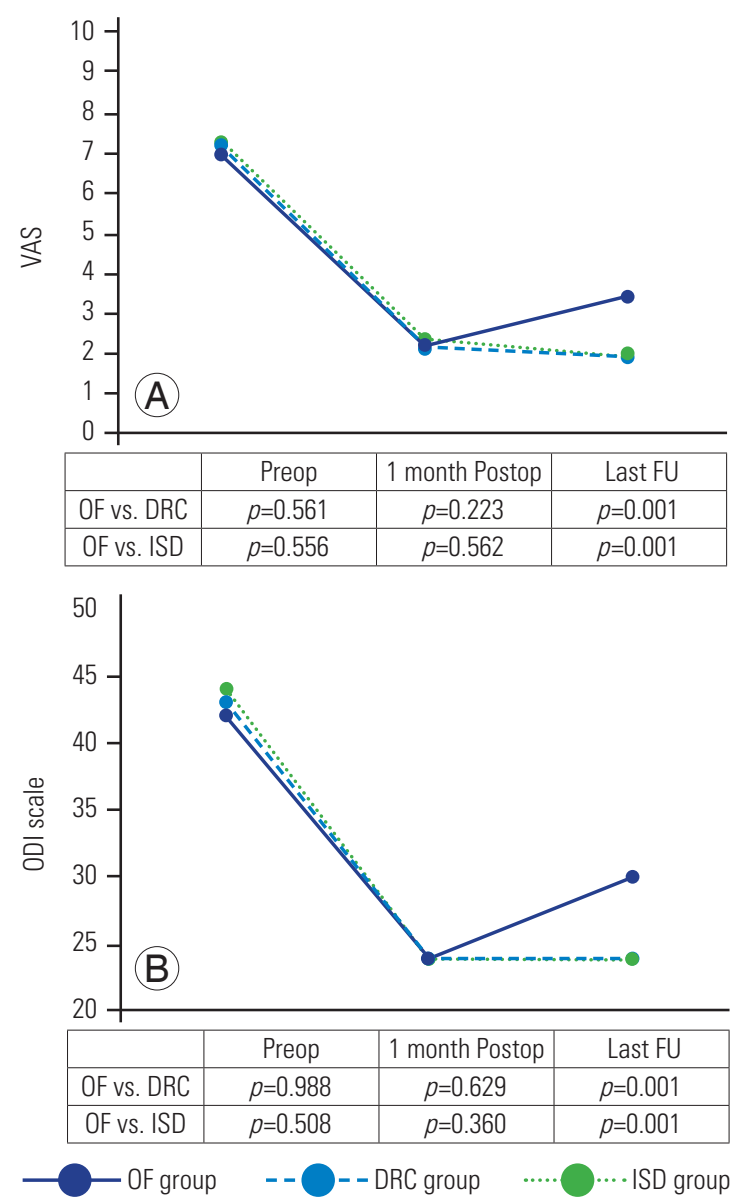

Fig. 4. (A) Median Visual Analog Scale (VAS) and (B) Oswestry Disability Index (ODI) measured prior to surgery, 1 month postoperative (Postop), and at last follow-up (FU), according to group of treatment: only fusion (OF group), dynamic rods construct (DRC group), and interspinous device (ISD group).

In this study, we analyzed the clinical and radiological results in a series of patients with single- and two-level lumbar spine degenerative disease, surgically treated with two different hybrid lumbar instrumentation techniques (DRC and ISD) for the prevention of ASDe. We compared those results with a series of patients surgically treated with lumbar-instrumented fusion without dynamic instrumentation. To the best of our knowledge, this study is one of the few trials to address a comparative analysis of whether or not to use the topping-off technique at adjacent level when lumbar fusion is intended to prevent the progression of degenerative changes at adjacent lumbar level and symptomatology associated to these changes.

Topping-off techniques refer to the application of instrumented non-fusion techniques that provide a transitional zone between fused segments and cephalad-mobile unfused levels [12,13]. Dynamic stabilization devices for

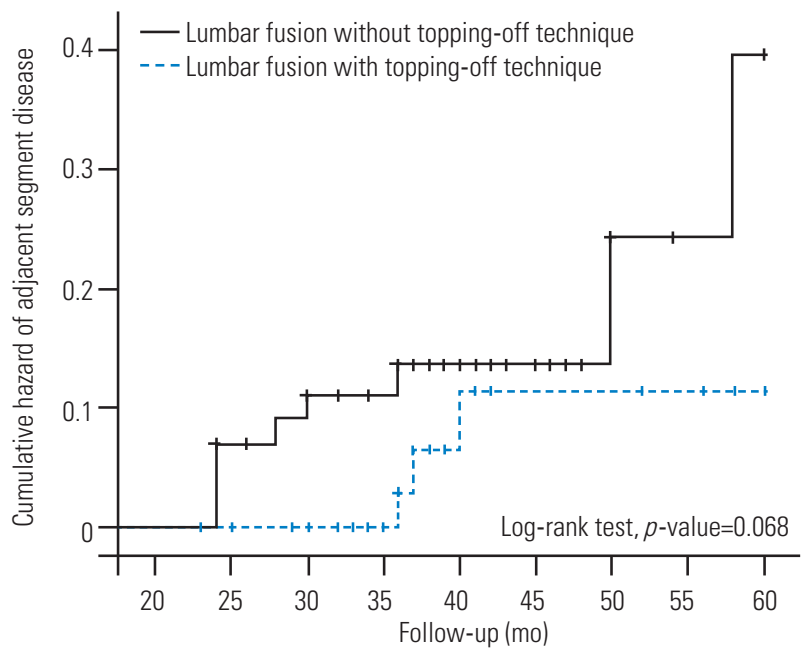

Fig. 5. Cumulative hazard function of adjacent segment disease (as defined in methods section) within 60 months after surgery according to use of a toppingoff technique such as dynamic rods constructs or interspinous device.

the topping-off technique are classified into three categories: (1) hybrid stabilization device with pedicle screw or rod construct, (2) interspinous process devices, and (3) total facet replacement system [14]. It has been demonstrated that dynamic techniques preserve, although limits motions in extension, flexion, and lateral bending, but do not completely compensate for increases in rotational movement as compared to that in the intact spine [15]. Intradiscal pressure at the adjacent level seems to be reduced; therefore, hybrid surgery might exert a preventive effect on degenerative disc changes [16]. When disc degeneration is already present at the adjacent segment, spinal surgeons must decide either to accept the risk of ASDe by operating only on index levels or to fuse all degenerated levels, with the risk of shifting the problem one level superiorly.

Even when in vitro studies clearly show that dynamic stabilization systems decrease stress loads to the adjacent level, there is no convincing evidence regarding the clinical and radiological benefits of the hybrid devices, with studies showing variable results with the topping-off technique [9,17-20]. Putzier et al. [9] reported radiological benefits of avoiding degeneration progression with dynamic instrumentation; however, they were unable to detect clinical differences in the outcome. Another study found that hybrid systems delayed but did not prevent ASDe [13]. Aygun et al. [21] used a cosmic pedicle screw rod system for a topping-off technique and found a limited role in preventing ASDi. Chen et al. [22] found that 
the topping-off technique with a Coflex ISD could not maintain disc height, neural foramen height, and width of adjacent segment during follow-up, with a decrease in the incidence of ASDi compared to that in the OF group. A meta-analysis showed that the difference in the incidence of ASDe or ASDi at the adjacent level was significant among the groups; the fusion group presented a significantly higher percentage of ASDi (11.6\% versus 5.1\%) and ASDe (52.6\% versus $12.6 \%$ ) as well as higher rates of revision surgery ( $8.1 \%$ versus $3 \%)$ as compared to that in the topping-off groups [23-25]. Another recent meta-analysis found that topping-off techniques can effectively prevent ASDe and ASDi from progressing after lumbar internal fixation, although they report important differences between the diagnostic criteria and clinical analysis; further, they report that this was more effective in improving the subjective feelings of the patients, similar to that in our findings [26]. A systematic review by Chou et al. [3] found that the OF group had a significantly higher incidence of ASDe and ASDi and a higher incidence of revision surgery. The World Federation of Neurosurgical Societies suggests that dynamic fusion constructs represent a treatment option that may help prevent ASD; however, there is no definite evidence of efficacy [27].

All the patients in our study had degenerative changes at adjacent level before the surgery; therefore, we fo- cused our analysis on the benefit of using the toppingoff technique for preventing ASDe progression and ASDi development. We had an overall ASDe progression rate of $68.3 \%$ with no significant difference between the OF and topping-off groups. However, we found that dynamic instrumentation had a protective effect against ASDi because both DRC and ISD groups had better clinical outcomes, with the postoperative ODI and VAS score
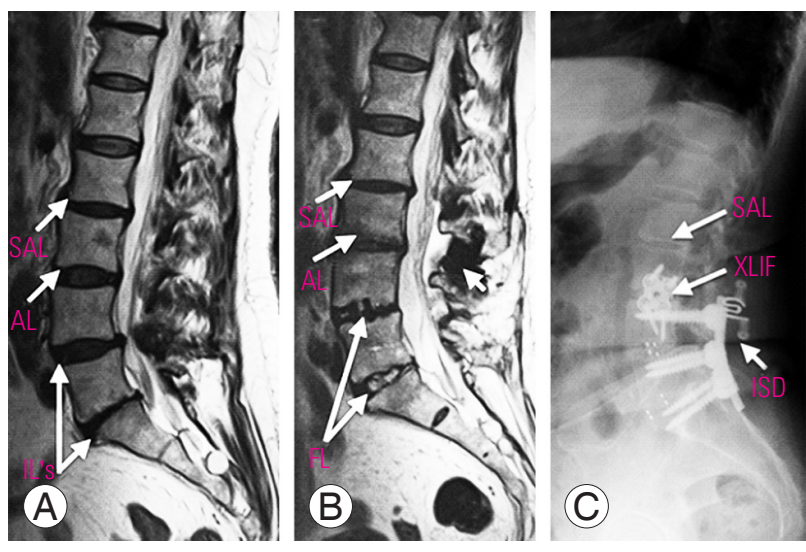

Fig. 6. Patient treated with topping-off technique with interspinous device (Stenofix) (A) that developed adjacent segment disease (B), and needed reoperation (C); an extreme lateral interbody fusion (XLIF) approach was made to treat adjacent segment disease. IL's, index levels; AL, adjacent level; SAL, supraadjacent level; DRC, dynamic rods construct; ISD, interspinous device; ASDe, adjacent segment degeneration.

Table 2. Logistic regression model and Cox regression model results considering ASDe and ASDi, respectively, as depending variable

\begin{tabular}{|c|c|c|}
\hline \multirow{2}{*}{ Variable } & Logistic regression model (ASDe) & Cox regression model (ASDi) \\
\hline & OR $(95 \% \mathrm{CI})$ & $\mathrm{HR}(95 \% \mathrm{CI})$ \\
\hline Age & 1.008 (0.969-1.048) & $1.029(0.963-1.1)$ \\
\hline Gender (female) & $1.442(0.54-3.849)$ & $2.915(0.442-19.222)$ \\
\hline Body mass index & $1.005(0.902-1.118)$ & $0.889(0.748-1.058)$ \\
\hline Smoker (yes) & $1.045(0.411-2.656)$ & $1.49(0.276-8.043)$ \\
\hline Pffirman preoperative classification of adjacent segment & $5.323(1.055-26.85)$ & $1.199(0.128-11.258)$ \\
\hline Preoperative L1-S1 Cobb angle & $1.005(0.942-1.073)$ & $0.942(0.853-1.041)$ \\
\hline Preoperative pelvic incidence & $0.811(0.364-1.806)$ & 1.191 (0.799-1.777) \\
\hline Preoperative pelvic tilt & $1.215(0.549-2.687)$ & $0.776(0.509-1.184)$ \\
\hline Preoperative sacral slope & $1.153(0.518-2.566)$ & $0.822(0.547-1.235)$ \\
\hline Preoperative UCLA classification of adjacent segment & $0.185(0.03-1.13)$ & $7.44(0.747-74.141)$ \\
\hline One-level fusion (vs. two-level fusion) & $0.996(0.393-2.525)$ & $2.233(0.581-8.592)$ \\
\hline Use of topping-off technique (DRC or ISD) & $1.229(0.458-3.303)$ & $0.154(0.031-0.773)$ \\
\hline
\end{tabular}

Statistically significant differences are shown in bold.

ASDe, adjacent segment degeneration; ASDi, adjacent segment disease (according to criteria defined in methods section); OR, odds ratio; $\mathrm{Cl}$, confidence interval; HR, hazard ratio; DRC, dynamic rods construct; ISD, interspinous device. 
being lower in both dynamic techniques than that in the OF group. ASDi was found in $12.8 \%$ of all the patients, with differences between groups as follows: $20 \%$ in the OF group, $4.2 \%$ in the DRC group, and $6.1 \%$ in the ISD group. These results contrast with those found in radiological parameters because all the groups had similar rates of ASDe. The decrease in the range of motion and intradiscal pressure provided with dynamic instrumentation at the adjacent segment could prevent ASDe changes from becoming symptomatic. The overall reoperation rate was $4.2 \%$ because most patients with ASDi had clinical improvement with conservative measures (Table 1, Fig. 6). We found no clinical or radiological differences between the DRC and ISD groups. Patients treated with both techniques had similar surgical results; both groups showed improved clinical outcomes and prevented ASDe from becoming symptomatic although both the techniques were unable to prevent ASDe progression as compared to the OF group.

Another important issue regarding the extension of the instrumentation one level above index segment with dynamic instrumentation is that the degenerative changes could develop in the supra-adjacent level. Hybrid stabilization systems could increase the stress at the supraadjacent level, thus accelerating the degenerative changes [28]. There was a significant decrease in the disc height and a significant progression in the UCLA scale at the supra-adjacent level in the ISD group as compared to that in the OF group (Fig. 3).

According to logistic regression model and Cox regression model, higher degenerative disc changes, as per the Pffirman classification [5], represent an independent risk factor associated with ASDe progression (Table 2); sex, BMI, smoking, or number of levels treated were not related to the study outcome. Use of topping-off instrumentation was a protective factor for the development of ASDi (Table 2).

There were two principal limitations of our study: the first was the retrospective study design. Further prospective studies are strongly recommended to corroborate our findings. Second, owing to the logistics at our institution, we performed postoperative MRI only in patients with a clinically worsening condition, and we performed our analyses using standardized radiological criteria for ASDe that can be observed using lumbar radiography.

\section{Conclusions}

Dynamic fixation is a safe procedure that is not associated with radiological improvement of the adjacent segment. However, we observed better clinical outcomes in terms of preventing symptomatic ASDi.

\section{Conflict of Interest}

No potential conflict of interest relevant to this article was reported.

\section{Author Contributions}

Salvador Fuster: senior author and revision; Jaime Jesús Martínez-Anda: clinical charters revision and article writing; Sergio Antonio Castillo-Rivera: clinical charters revision; Caribay Vargas-Reverón: article writing; and Eduard Tornero: revision, statistics, and corresponding author.

\section{References}

1. Chen BL, Wei FX, Ueyama K, Xie DH, Sannohe A, Liu SY. Adjacent segment degeneration after single-segment PLIF: the risk factor for degeneration and its impact on clinical outcomes. Eur Spine J 2011;20:1946-50.

2. Helgeson MD, Bevevino AJ, Hilibrand AS. Update on the evidence for adjacent segment degeneration and disease. Spine J 2013;13:342-51.

3. Chou PH, Lin HH, An HS, Liu KY, Su WR, Lin CL. Could the topping-off technique be the preventive strategy against adjacent segment disease after pedicle screw-based fusion in lumbar degenerative diseases?: a systematic review. Biomed Res Int 2017;2017:4385620.

4. Ghiselli G, Wang JC, Hsu WK, Dawson EG. L5-S1 segment survivorship and clinical outcome analysis after L4-L5 isolated fusion. Spine (Phila Pa 1976) 2003;28:1275-80.

5. Pfirrmann CW, Metzdorf A, Zanetti M, Hodler J, Boos N. Magnetic resonance classification of lumbar intervertebral disc degeneration. Spine (Phila $\mathrm{Pa}$ 1976) 2001;26:1873-8.

6. Fairbank JC, Pynsent PB. The Oswestry Disability Index. Spine (Phila Pa 1976) 2000;25:2940-52.

7. Modic MT, Steinberg PM, Ross JS, Masaryk TJ, 
Carter JR. Degenerative disk disease: assessment of changes in vertebral body marrow with MR imaging. Radiology 1988;166(1 Pt 1):193-9.

8. Okuda S, Oda T, Miyauchi A, et al. Lamina horizontalization and facet tropism as the risk factors for adjacent segment degeneration after PLIF. Spine (Phila Pa 1976) 2008;33:2754-8.

9. Putzier M, Hoff E, Tohtz S, Gross C, Perka C, Strube P. Dynamic stabilization adjacent to single-level fusion: part II. No clinical benefit for asymptomatic, initially degenerated adjacent segments after 6 years followup. Eur Spine J 2010;19:2181-9.

10. Yang JY, Lee JK, Song HS. The impact of adjacent segment degeneration on the clinical outcome after lumbar spinal fusion. Spine (Phila Pa 1976) 2008;33:503-7.

11. Bono CM, Lee CK. Critical analysis of trends in fusion for degenerative disc disease over the past 20 years: influence of technique on fusion rate and clinical outcome. Spine (Phila Pa 1976) 2004;29:455-63.

12. Zhu Z, Liu C, Wang $K$, et al. Topping-off technique prevents aggravation of degeneration of adjacent segment fusion revealed by retrospective and finite element biomechanical analysis. J Orthop Surg Res 2015;10:10.

13. Kashkoush A, Agarwal N, Paschel E, Goldschmidt E, Gerszten PC. Evaluation of a hybrid dynamic stabilization and fusion system in the lumbar spine: a 10 year experience. Cureus 2016;8:e637.

14. Khoueir P, Kim KA, Wang MY. Classification of posterior dynamic stabilization devices. Neurosurg Focus 2007;22:E3.

15. Beastall J, Karadimas E, Siddiqui M, et al. The Dynesys lumbar spinal stabilization system: a preliminary report on positional magnetic resonance imaging findings. Spine (Phila Pa 1976) 2007;32:685-90.

16. Cabello J, Cavanilles-Walker JM, Iborra M, Ubierna MT, Covaro A, Roca J. The protective role of dynamic stabilization on the adjacent disc to a rigid instrumented level: an in vitro biomechanical analysis. Arch Orthop Trauma Surg 2013;133:443-8.

17. Lee CH, Jahng TA, Hyun SJ, et al. Dynamic stabilization using the Dynesys system versus posterior lumbar interbody fusion for the treatment of degenerative lumbar spinal disease: a clinical and radiological outcomes-based meta-analysis. Neurosurg Focus 2016;40:E7.
18. Baioni A, Di Silvestre M, Greggi T, Vommaro F, Lolli F, Scarale A. Does hybrid fixation prevent junctional disease after posterior fusion for degenerative lumbar disorders?: a minimum 5-year follow-up study. Eur Spine J 2015;24 Suppl 7:855-64.

19. Maserati MB, Tormenti MJ, Panczykowski DM, Bonfield CM, Gerszten PC. The use of a hybrid dynamic stabilization and fusion system in the lumbar spine: preliminary experience. Neurosurg Focus 2010;28:E2.

20. Herren C, Sobottke R, Pishnamaz M, et al. The use of the DTO(TM) hybrid dynamic device: a clinical outcome- and radiological-based prospective clinical trial. BMC Musculoskelet Disord 2018;19:199.

21. Aygun H, Yaray O, Mutlu M. Does the addition of a dynamic pedicle screw to a fusion segment prevent adjacent segment pathology in the lumbar spine? Asian Spine J 2017;11:715-21.

22. Chen XL, Guan L, Liu YZ, Yang JC, Wang WL, Hai Y. Interspinous dynamic stabilization adjacent to fusion versus double-segment fusion for treatment of lumbar degenerative disease with a minimum follow-up of three years. Int Orthop 2016;40:1275-83.

23. Kumar MN, Baklanov A, Chopin D. Correlation between sagittal plane changes and adjacent segment degeneration following lumbar spine fusion. Eur Spine J 2001;10:314-9.

24. Aota Y, Kumano K, Hirabayashi S. Postfusion instability at the adjacent segments after rigid pedicle screw fixation for degenerative lumbar spinal disorders. J Spinal Disord 1995;8:464-73.

25. Lee CS, Hwang CJ, Lee SW, et al. Risk factors for adjacent segment disease after lumbar fusion. Eur Spine J 2009;18:1637-43.

26. Wang W, Sun X, Zhang T, et al. Comparison between topping-off technology and posterior lumbar interbody fusion in the treatment of chronic low back pain: a meta-analysis. Medicine (Baltimore) 2020;99:e18885.

27. Roitberg B, Zileli M, Sharif S, Anania C, Fornari M, Costa F. Mobility-preserving surgery for lumbar spinal stenosis: WFNS Spine Committee recommendations. World Neurosurg X 2020;7:100078.

28. Lu K, Liliang PC, Wang $\mathrm{HK}$, et al. Reduction in adjacent-segment degeneration after multilevel posterior lumbar interbody fusion with proximal DIAM implantation. J Neurosurg Spine 2015;23:190-6. 